\title{
Dynamics of the quantum dimer model on the triangular lattice: Soft modes and local resonating valence-bond correlations
}

\author{
Arnaud Ralko, ${ }^{1}$ Michel Ferrero, ${ }^{2}$ Federico Becca, ${ }^{1,2}$ Dmitri Ivanov, ${ }^{1}$ and Frédéric Mila ${ }^{1}$ \\ ${ }^{1}$ Institute of Theoretical Physics, Ecole Polytechnique Fédérale de Lausanne (EPFL), CH-1015 Lausanne, Switzerland \\ ${ }^{2}$ INFM-Democritos, National Simulation Center and International School for Advanced Studies (SISSA), I-34014 Trieste, Italy
}

(Received 24 July 2006; published 9 October 2006)

\begin{abstract}
We report on an exhaustive investigation of the dynamical dimer-dimer correlations in imaginary time for the quantum dimer model on the triangular lattice using the Green's function Monte Carlo method. We show in particular that soft modes develop upon reducing the dimer-dimer repulsion, indicating the presence of a second-order phase transition into an ordered phase with broken translational symmetry. We further investigate the nature of this ordered phase, for which a 12-site unit cell has been previously proposed, with the surprising result that significant Bragg peaks are only present at two of the three high-symmetry points consistent with this unit cell. We attribute the absence of a detectable peak to its small magnitude due to the nearly uniform internal structure of the 12-site crystal cell.
\end{abstract}

DOI: 10.1103/PhysRevB.74.134301

PACS number(s): 05.50.+q, 05.30.-d, 75.10.Jm

\section{INTRODUCTION}

Resonating valence-bond (RVB) states constitute a major theme in strongly correlated systems, both in the context of Mott insulators and of superconductors. ${ }^{1,2}$ In his milestone paper, Anderson proposed that high-temperature superconductors are intimately related to Mott insulators and that the pairing mechanism is due to "preformed" pairs already present in the strongly correlated insulating phase. ${ }^{1}$ Originally, the RVB phase was defined by a fully projected BCS wave function where the electron pairs have an arbitrary range. A simplification of the RVB construction was further proposed by Rokhsar and Kivelson, who considered an effective quantum dimer model (QDM) with only local processes and orthogonal dimer coverings. ${ }^{3}$ The conditions under which such a Hamiltonian could be an accurate description of an SU(2) Heisenberg model are not fully understood yet, but it is expected to be a reasonable approximation if the dimer coverings constitute a natural variational basis, and specific quantum dimer models have recently been derived for the trimerized Kagome antiferromagnet ${ }^{4}$ by Zhitomirsky, ${ }^{5}$ and for a spin-orbital model by Vernay and collaborators. ${ }^{6}$

Regardless of its actual validity for microscopic spin models, the QDM of Rokhsar and Kivelson has attracted a lot of attention as a promising way to investigate RVB physics. In particular, the QDM on the triangular lattice has been shown to possess a liquid phase with an exponential decay of all correlations. ${ }^{7}$ The Hamiltonian of that model is

$$
\begin{aligned}
H & =-t \sum(|/ /\rangle\langle-|+\text { H.c. }) \\
& +V \sum\left(|/ /\rangle\left\langle/ /|+| \_\right\rangle\langle-|\right),
\end{aligned}
$$

where the sum runs over all plaquettes including the three possible orientations. The kinetic term controlled by the amplitude $t$ changes the dimer covering of every flippable plaquette, i.e., of every plaquette containing two dimers facing each other, while the potential term controlled by the interaction $V$ describes a repulsion $(V>0)$ or an attraction $(V<0)$ between dimers facing each other.

Using the Green's function Monte Carlo (GFMC) algorithm to probe the ground-state properties of large clusters, we recently proved the existence, in the thermodynamic limit, of topological degeneracy in the disordered (i.e., RVB) phase. ${ }^{8}$ This degeneracy persists over an extended parameter range below the Rokhsar-Kivelson point $V / t=1$. With decreasing $V / t$, the RVB phase is replaced by a crystalline phase with a 12 -site unit cell, the so-called $\sqrt{12} \times \sqrt{12}$ phase. Upon further decreasing the dimer-dimer repulsion, this phase is ultimately followed by a columnar phase for sufficiently large and negative $V / t$.

From the point of view of RVB physics, one of the most interesting questions is the nature of the crystallization transition between the RVB and the $\sqrt{12} \times \sqrt{12}$ phases. In the present work, we analyze this phase transition by extending the GFMC algorithm to calculate dynamical dimer-dimer correlations, as done in Ref. 9 at the Rokhsar-Kivelson point with a simpler algorithm. This has allowed us to extract the energy of the first dimer excitation throughout the Brillouin zone (BZ). In the RVB phase, we expect this dimer excitation branch to have a gap. ${ }^{10}$ Having in mind that the $\sqrt{12}$ $\times \sqrt{12}$ phase has dimer order in the thermodynamic limit, this gap must close at some $k$ points of the $\mathrm{BZ}$ upon entering that phase. An analysis of the gap behavior (on the disordered side) and of the structure factor (on the ordered side) near the transition point gives a strong evidence in favor of a second order phase transition.

The paper is organized as follows. In Sec. II, we give a brief explanation of how we calculate the dynamical correlations and discuss the importance of the choice of the finitesize clusters. Then, in Sec. III we discuss the excitation spectrum, as well as the location and the nature of the phase transition between the RVB liquid and the crystalline phase. Finally, Sec. IV is dedicated to the internal structure of the $\sqrt{12} \times \sqrt{12}$ phase. Concluding remarks are given in Sec. V. 


\section{THE NUMERICAL METHOD}

The numerical investigations are based on a zerotemperature Monte Carlo method that filters out the highenergy components of a starting wave function $\left|\Psi_{G}\right\rangle$ by the iterative application of $G=(\Lambda-H)$, where $\Lambda$ is a diagonal matrix proportional to the identity. This allows us to directly sample the ground state $\left|\Psi_{0}\right\rangle$. Indeed, in the case of the QDM defined by Eq. (1), all the off-diagonal matrix elements are nonpositive and, therefore, there is no sign problem and the GFMC is numerically exact. ${ }^{11}$ Moreover, in addition to the ground-state energy and static correlation functions, it is also possible within the same method to compute dynamical correlations in imaginary time, such as the dimer-dimer correlation function defined by

$$
D(k, \tau)=\frac{\left\langle\Psi_{G}\left|d(-k) e^{-H \tau} d(k)\right| \Psi_{0}\right\rangle}{\left\langle\Psi_{G}\left|e^{-H \tau}\right| \Psi_{0}\right\rangle},
$$

where $d(k)$ is the Fourier transform of the dimer operator $d(r)$ in real space that is equal to 1 if there is a dimer at site $r$ in the $(1,0)$ direction and 0 otherwise. The expression of Eq. (2) can be easily computed by GFMC by applying the so-called forward-walking technique. ${ }^{12}$ This method to calculate dynamic correlation functions has been recently used to investigate an $X Y$ spin model with ring exchange. ${ }^{13}$ In practice, after an equilibration time, the dimer configurations $|x\rangle$ are statistically sampled. Then the (diagonal) dimer operator $d(k)$ is applied to $|x\rangle$ and the system is evolved for $L$ further steps by the forward walking. Within this technique, it is very important to analytically perform the limit of $\Lambda$ $\rightarrow \infty,{ }^{14}$ which allows us to speed up the algorithm and define the imaginary time $\tau$. In more detail, in the limit $\Lambda \rightarrow \infty$, the probability of the diagonal moves grows and, therefore, it is better to sample directly the number of diagonal moves before changing the configuration $|x\rangle$. Note that, in order to have a finite probability for non-diagonal moves for $\Lambda \rightarrow \infty$, we have to apply $G$ to a power proportional to $\Lambda$, i.e., $G^{\beta \Lambda}$. Then

$$
\lim _{\Lambda \rightarrow \infty}(\Lambda-H)^{\beta \Lambda} \sim e^{-H \beta}
$$

This algorithm allows us to perform at once all the diagonal moves and then perform directly the nondiagonal one, thus saving a huge computational effort. Then

$$
D(k, L \beta)=\lim _{M \rightarrow \infty} \frac{\left\langle\Psi_{G}\left|d(-k) e^{-H L \beta} d(k) e^{-H M \beta}\right| \Psi_{G}\right\rangle}{\left\langle\Psi_{G}\left|e^{-H(L+M) \beta}\right| \Psi_{G}\right\rangle},
$$

and the imaginary time of Eq. (2) is given by $\tau=L \beta$. It is easy to demonstrate that for large $\tau$, when all the high-energy states have been projected out, we have

$$
D(k, \tau) \propto e^{-\Delta(k) \tau} .
$$

Then, by fitting the large- $\tau$ behavior of $D(k, \tau)$, it is possible to estimate the gap $\Delta(k)$ of the first excited state at the $k$ point in the BZ. Note that, within this approach, we only find the excitations that have a finite overlap with the state $d(k)\left|\Psi_{0}\right\rangle$. In this work, we are only interested in the lowest excitation energy, which can be extracted by considering only one exponential and fitting the largest- $\tau$ regime. In or-

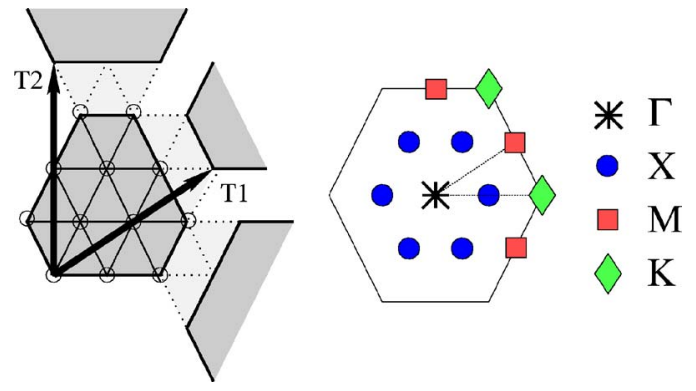

FIG. 1. (Color online) Left: Example of cluster used in the calculations (here the 12-site one). Right: Brillouin zone of the triangular lattice. High-symmetry points are labeled by $K=(4 \pi / 3,0)$, $M=(\pi, \pi / \sqrt{3})$, and $X=(2 \pi / 3,0)$.

der to verify the accuracy on $\Delta(k)$, we have also checked the results by fitting with two and three exponentials. For extracting more information on the spectrum, one may implement more involved methods such as the maximum-entropy technique, ${ }^{15}$ which goes beyond the scope of the present paper.

Since we are interested in the transition between the disordered RVB phase and the crystalline $\sqrt{12} \times \sqrt{12}$ one, we have decided to work only with clusters defined by factorization of the infinite triangular lattice by the translations ${ }^{16}$

$$
\begin{gathered}
\mathbf{T}_{1}=l \mathbf{u}_{1}+l \mathbf{u}_{2}, \\
\mathbf{T}_{2}=-l \mathbf{u}_{1}+2 l \mathbf{u}_{2},
\end{gathered}
$$

where $\mathbf{u}_{1}=(1,0)$ and $\mathbf{u}_{2}=(1 / 2, \sqrt{3} / 2)$ are the unitary vectors defining the triangular lattice and $l$ is an integer. Such clusters can accommodate the proposed crystal structure without defects. The number of sites in the cluster is $N=3 l^{2}$, and to admit dimer coverings $l$ must be even. An example of such a cluster for $l=2$ is shown in Fig. 1. The right panel of Fig. 1 shows the BZ of the triangular lattice with the highsymmetry points $K=(4 \pi / 3,0), \quad M=(\pi, \pi / \sqrt{3})$, and $X$ $=(2 \pi / 3,0)$.

We remind the reader that the QDM on a toroidal cluster has four topological sectors denoted by $(0,0),(1,0),(0,1)$, and $(1,1)$ : The Hilbert space splits into four subspaces that cannot be connected by local dimer flips such as the ones contained in the Hamiltonian. Given the rotational symmetry of the cluster, three of the four topological sectors are always exactly degenerate, and the only independent ones are the $(0,0)$ and the $(1,1)$, in the notation of Ref. 8 . The ground state belongs to the former or the latter depending on the parity of $l / 2{ }^{8}$ It is worthwhile to mention that, within GFMC, we can fix the topological sector during the simulation and, therefore, we can study the different sectors independently.

In the following, we will present the results for the dimer gap along particular symmetry directions of the BZ, and in particular the size scaling of the $k$ points where soft modes are expected to develop upon entering the crystalline $\sqrt{12} \times \sqrt{12}$ phase. 


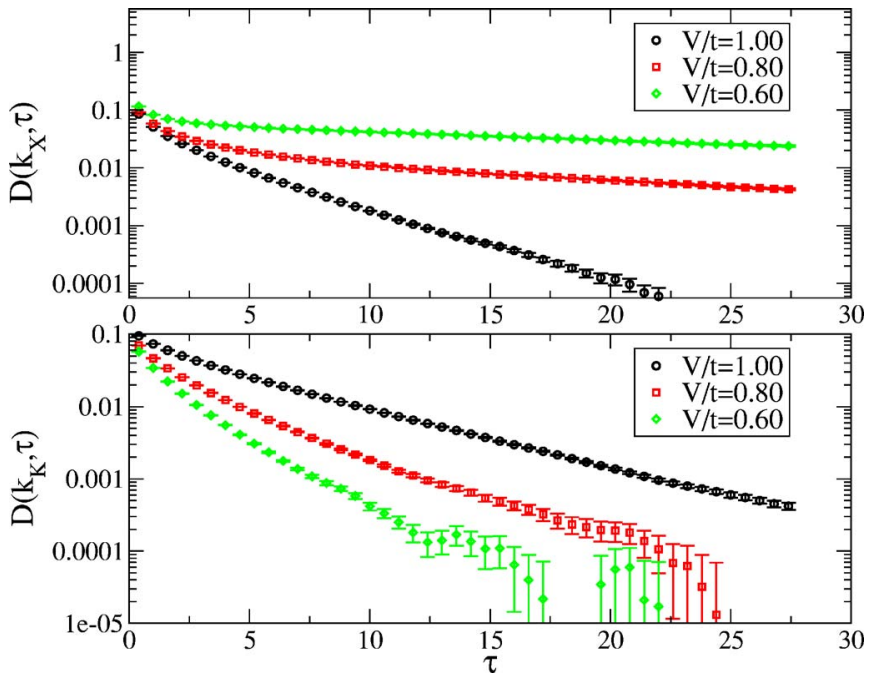

FIG. 2. (Color online) Typical results for the dynamical dimerdimer correlations $D(k, \tau)$ for the 432-site cluster at $X=(2 \pi / 3,0)$ (upper panel) and $K=(4 \pi / 3,0)$ (lower panel) for a few selected values of $V / t$.

\section{THE RVB-CRYSTAL TRANSITION}

In their original work, Moessner and Sondhi ${ }^{7}$ estimated that the transition from the disordered to a crystalline phase occurred around $V / t \simeq 0.7$. A later analysis of the topological splitting on finite-size clusters suggested a slightly larger value $(0.7<V / t<0.85){ }^{8}$ The analysis of dynamic correlations reported in our present work provides a more accurate estimate $V / t=0.82 \pm 0.03$, as will be demonstrated below.

In the crystalline phase, the existence of a broken translational symmetry naturally implies a folding of the BZ and soft modes at particular $k$ points. In particular, for the 12 -site unit cell proposed by Moessner and Sondhi, we expect to find soft modes at the $X, M$, and $K$ points (see Fig. 1). So we now turn to an investigation of the excitation spectrum as revealed by our GFMC approach, with emphasis on the behavior around these high symmetry points.

We perform a systematic analysis of the dynamical correlations for clusters with up to 432 sites and for $0.55 \leqslant V / t$ $\leqslant 1.0$. We report in Fig. 2 the results for a 432-site cluster $\left(N=3 l^{2}\right.$ with $\left.l=12\right)$ at $X$ and $K$ for different values of $V / t$. Although in principle the long-time dynamics should always give access to the energy of the first excited states, in practice the error bars grow with time, and two types of data sets may be distinguished. In the first, favorable case, an exponential decay governed by a single exponent shows up long before the error bars become significant, and the gap can be extracted very accurately (see upper panel of Fig. 2). In a logarithmic scale, this shows up as a linear behavior of $D(k, \tau)$ prior to the development of large error bars. This is the case throughout the BZ at the Rokhsar-Kivelson point $(V / t=1)$, and everywhere except around the $K$ point when $V / t<1$. In the other case, the error bars start to grow before a simple exponential decay has set in. In logarithmic scale, this shows up as a persistent curvature. An example is reported in the lower panel of Fig. 2. The absence of a clear exponential regime prevents us from extracting a reliable

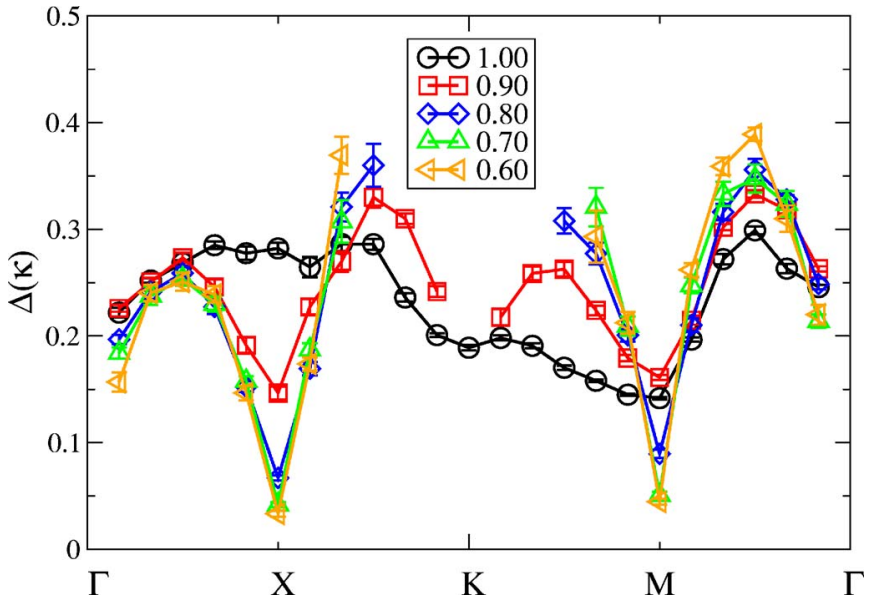

FIG. 3. (Color online) Lowest-energy excitation spectra for the 432-site cluster for different values of $V / t$.

value for the energy of the first excited state.

The results of this analysis are depicted in Fig. 3, where we show the excitation spectrum of the 432-site cluster in the topological sector $(0,0)$ (the one that contains the ground state) for different values of $V / t$. The missing points around $K$ correspond to cases where the extraction of the energy was impossible. This spectrum calls for several comments.

First of all, the spectrum is fully gapped close to the Rokhsar-Kivelson point, in agreement with the results obtained previously by Ivanov at the Rokhsar-Kivelson point, ${ }^{9}$ and consistent with a liquid ground state with no long-range order. Secondly, upon decreasing $V / t$, two soft modes clearly appear at $X$ and $M$. These two $k$ points are compatible with the 12 -site unit cell structure of the $\sqrt{12} \times \sqrt{12}$ phase, proposed by Moessner and Sondhi. ${ }^{17}$ It turns out that the numerical results concerning $X$ and $M$ are very accurate and allow us to obtain a rather precise determination of the transition point. In Fig. 4, we show the size scaling of these two gaps. At the Rokhsar-Kivelson point, the curves saturate

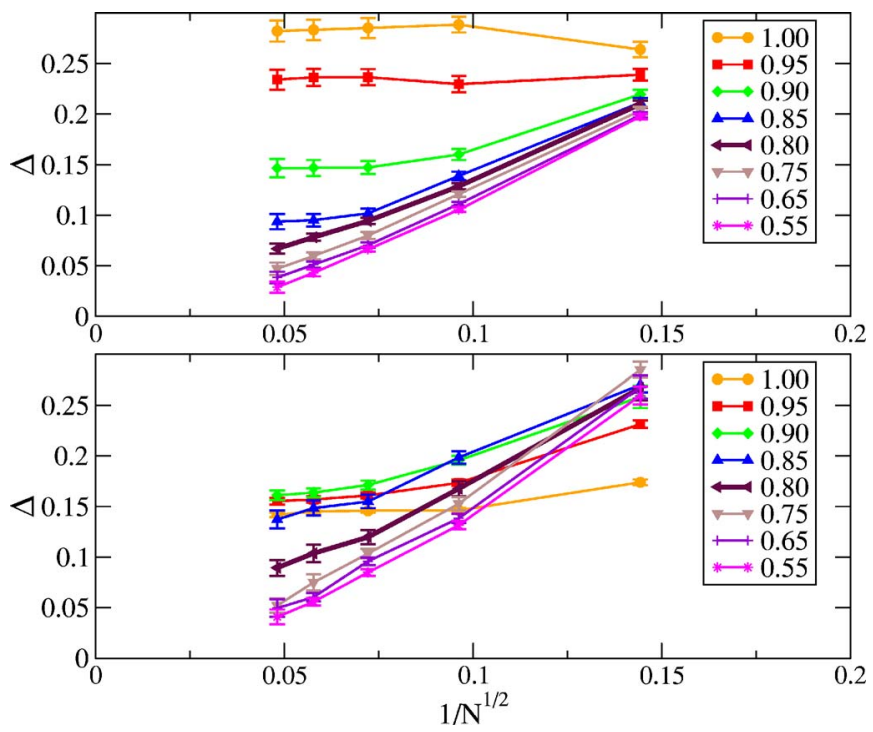

FIG. 4. (Color online) Size scalings of the gap at the $X$ (upper panel) and $M$ (lower panel) points for different values of $V / t$. 
quickly to a finite value in the thermodynamic limit. This value becomes smaller by decreasing the ratio $V / t$, and we can estimate that the transition occurs around $V / t \simeq 0.8$, not far from our previous estimate based on the topological gap. ${ }^{8}$ This constitutes one of the important results of this paper: The transition out of the RVB phase is second order, as revealed by the development of soft modes upon entering the crystalline phase.

Thirdly, and quite surprisingly, we could not get any evidence for the emergence of a soft mode at the $K$ point, the third high-symmetry point compatible with the 12 -site unit cell. On the contrary, the slope of $D(K, \tau)$ (in logarithmic scale) at the largest accessible times is still very large, so that there is no evidence whatsoever of a small energy scale at that point. We can think of two possibilities to explain this result. (i) The overlap between the first excitation and the starting state $d(k)\left|\Psi_{0}\right\rangle$ is so small that this excitation does not give any detectable contribution to the dynamics in the accessible time range. (ii) The symmetry of the ordered phase is such that no soft mode develops at the $K$ point. To clarify this point, we now turn to a detailed investigation of this crystalline phase.

\section{THE $\sqrt{12} \times \sqrt{12}$ PHASE}

The unexpected behavior found at $K$ is actually not in contradiction with any of the previous numerical studies of the static correlations in that phase: Moessner and Sondhi detected a peak at $X$ and noticed it was compatible with a 12 -site unit cell, but they did not calculate the full $k$ dependence of the static dimer-dimer correlations. ${ }^{7}$ In our previous paper, ${ }^{8}$ we concentrated on the RVB phase and showed that the correlations were indeed decreasing very fast in that phase, but we did not investigate in details the correlations in the ordered phase. So the first thing to do is to go back to the static correlations and to calculate the full $k$ dependence of the static dimer-dimer correlation function defined by

$$
D_{\text {stat }}(k)=\frac{\left\langle\Psi_{0}|d(-k) d(k)| \Psi_{0}\right\rangle}{\left\langle\Psi_{0} \mid \Psi_{0}\right\rangle}
$$

The results for different cluster sizes and $V / t=0.55$ are depicted in Fig. 5. Clearly, these results confirm the anomaly of the $K$ point: While strong peaks are present at $X$ and $M$ and grow with the size of the cluster, no peak could be detected at $K$. Notice that for this kind of static correlations, there is no fitting procedure, in contraxt to the extraction of the gap in the dynamical calculations.

Before trying to understand what is going on at the $K$ point, let us look more closely at the $X$ and $M$ points. For small enough $V / t$, the size of these peaks grows linearly with the number of sites of the cluster, a clear indication that Bragg peaks develop. Quantitatively, we extract the square of the order parameter defined by

$$
D_{k}^{2}=\frac{D_{\text {stat }}(k)}{N},
$$

for both $X$ and $M$ from the finite-size scaling of these peaks. The results are depicted in Fig. 5. The extrapolated values

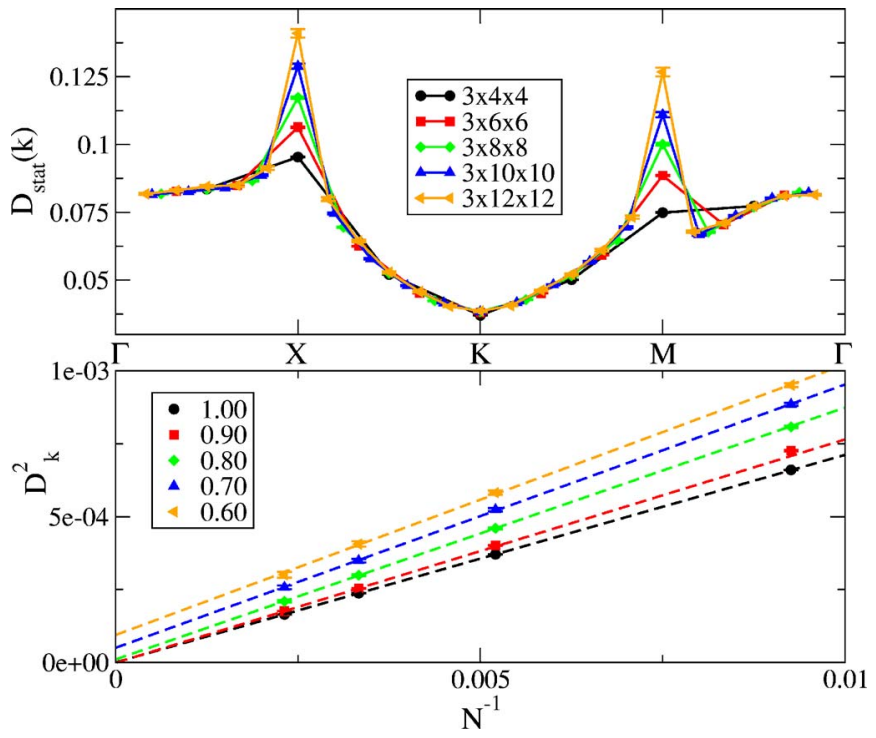

FIG. 5. (Color online) Static dimer-dimer correlation at $V / t$ $=0.55$ for different cluster sizes (upper panel). Size scaling of the dimer order parameter square $D_{k}^{2}$ (calculated at the $X$ point) for different values of $V / t$ (lower panel).

are very small even well inside the crystalline phase, but given the accuracy of the data and the smoothness of the size scaling, these values are reliable, and indeed they again point to a second-order phase transition located between $\mathrm{V} / \mathrm{t}$ $\simeq 0.8$ and 0.85 where the order parameters continuously vanish at both points (see Fig. 6). So the existence of a crystalline phase with Bragg peaks at $X$ and $M$ is well established.

A similar analysis of the Bragg peak at the $K$ point gives a zero value for $D_{k}^{2}$, within the error bars. For example, well inside the crystal phase at $V / t=0.55$ we can estimate an upper bound $D_{k}^{2}<1.5 \times 10^{-6}$.

As mentioned at the end of the previous section, there are two possible explanations for the absence of the $K$ peak. The first possibility is an exact cancellation of the $K$ peak due to the symmetry of the crystal phase. The second one is just a strong suppression of the $K$ peak so that its magnitude is below our numerical precision.

The symmetry cancellation of the $K$ peak might occur in a crystal phase with a threefold rotational symmetry about a

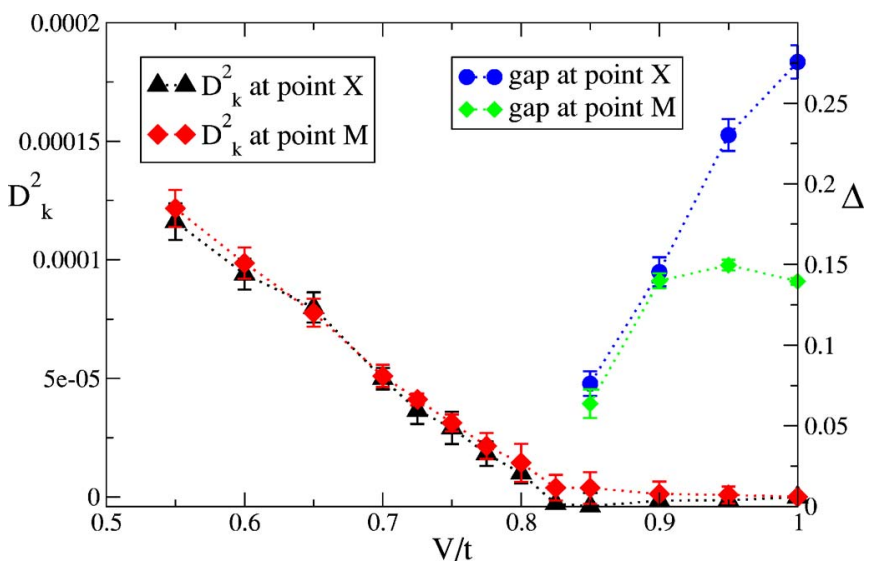

FIG. 6. (Color online) $V / t$ dependence of the thermodynamic values of $D_{k}^{2}$ and of the gap at the $X$ and $M$ points. 


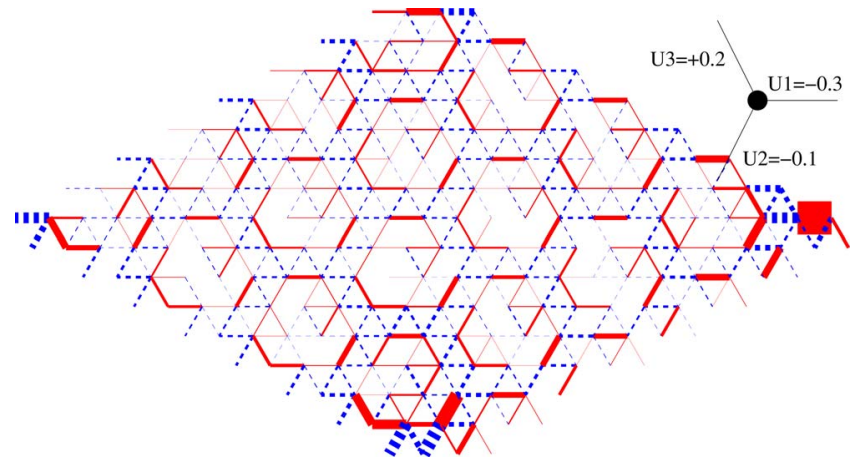

FIG. 7. (Color online) Mean value of the dimer operator for the 192 -site cluster for $V / t=0.6$ in the presence of the local perturbation (shown in the inset) placed at the rightest site. Dashed lines correspond to correlations lower than the uniform distribution (i.e., $1 / 6)$ and continuous lines to correlations larger than $1 / 6$. The thickness of the bonds indicates how different the amplitude is from the uniform distribution.

lattice site. ${ }^{18}$ However, this type of crystal symmetry seems to be unlikely, since it cannot accommodate any individual dimer or plaquette covering with the symmetry of the crystal (due to the single site in the center of the threefold symmetry). Note that the $\sqrt{12} \times \sqrt{12}$ crystal structure proposed by Moessner and Sondhi ${ }^{17}$ has a threefold symmetry about a triangular plaquette center and should not lead to an exact cancellation of the $K$ peak. To get a further insight in the symmetry of the $\sqrt{12} \times \sqrt{12}$ phase, we have calculated the static density profile in the presence of a local perturbation breaking all symmetries of the lattice. In this way, instead of obtaining a uniform linear combination of all symmetry related crystal states, the system selects one (or sometimes several) crystal state(s) favored by the perturbation, giving access to a real-space snapshot of the broken-symmetry ground state far enough from the perturbation. A typical result obtained by considering local potentials for three bonds related by a $2 \pi / 3$ rotation around a site that favor or disfavor the presence of a dimer is depicted in Fig. 7. The 12-site diamond of the type proposed by Moessner and Sondhi ${ }^{17}$ is clearly visible. Other choices of potential perturbation sometimes produce less symmetric periodic patterns which we interpret as superpositions of several crystal states. However, in no pattern were we able to detect a threefold symmetry about a lattice site. Therefore we conclude that the symmetry-cancellation scenario is not realized and that we should interpret the absence of a visible $K$ peak as a strong suppression of the corresponding Fourier harmonic in the $\sqrt{12} \times \sqrt{12}$ state of Moessner and Sondhi. ${ }^{17}$

Interestingly enough, while the unit cell is clearly visible in Fig. 7, there is no internal modulation of the dimer density inside the resonating 12-site hexagons. Motivated by these observations, we have performed a calculation with a trial wave function containing a uniform RVB superposition of all dimer configurations within 12-site hexagons and no dimers between the hexagons. The resulting peak weights $D_{X}^{2}$ and $D_{M}^{2}$ are of the same order of magnitude, while the peak weight $D_{K}^{2}$ is more than two orders of magnitude smaller. Such a huge difference between the peak weights would ex-

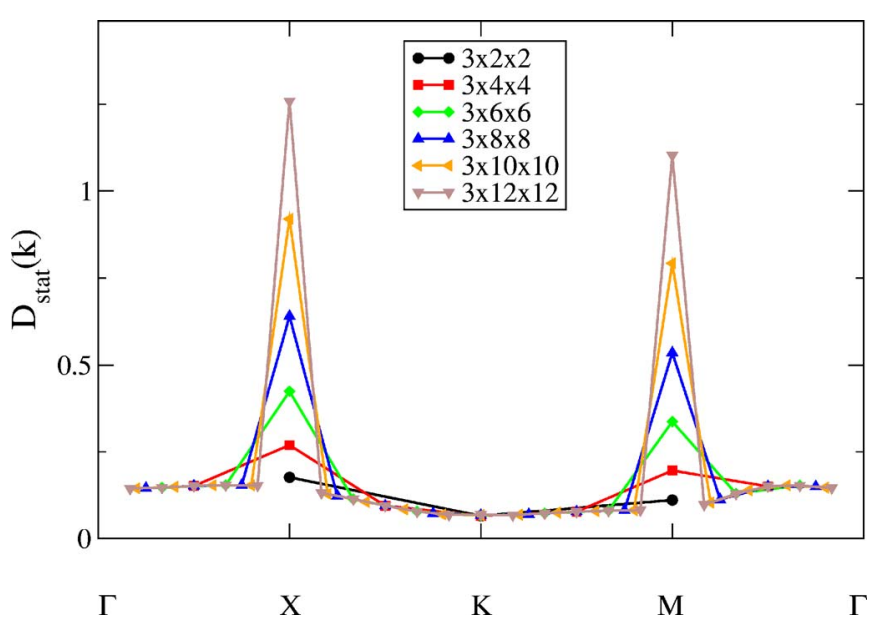

FIG. 8. (Color online) Static dimer correlations in the trial wave function constructed as the product of uniform RVB superpositions of dimer covering of 12-site hexagons.

plain our numerical findings about the absence of the $K$ peak for both dynamic and static correlations. For illustration, the static dimer-dimer correlations for our trial wave function are shown in Fig. 8: They qualitatively resemble the actual correlations in the crystal phase of Fig. 5, with no visible $K$ peak.

\section{DISCUSSION}

To summarize, we have studied the phase transition between the RVB and the crystal states of the QDM on the triangular lattice by analyzing the dimer excitation spectrum with the help of the Green's function Monte Carlo method. First of all, we have shown that upon approaching the transition between the RVB phase and the ordered phase, two soft modes develop in the ordered phase, while two Bragg peaks continuously disappear in the RVB phase. The continuous behavior of the dimer gap and of the Bragg peaks at the transition point strongly suggests the second order of the phase transition, consistent with the theory of Moessner and Sondhi. ${ }^{17}$ Furthermore, our analysis provides a more accurate location of the transition point $V / t=0.82 \pm 0.03$ than in previous studies.

A peculiar feature of the crystal phase is the apparent absence of a singularity (Bragg peak or soft mode) at the zone corner. We interpret this effect as a strong reduction of this Fourier harmonics in the crystal without much shortlength structure in the large 12-site crystal cell. In other words, the $\sqrt{12} \times \sqrt{12}$ crystal may be better approximated by 12 -site hexagons without internal structure, and not by resonating 4-site plaquettes.

It is interesting to compare these new findings with the phase diagram of the same model on the square lattice. In that case, the Rokhsar-Kivelson state realized at the RokhsarKivelson point $(V / t=1)$ is immediately replaced by a plaquette phase, until columnar dimmer order is finally stabilized for small enough repulsion. The plaquette state is a local RVB state on a square plaquette, and in that sense, it is similar to the local RVB state on the 12-site diamond. But 
the analogy can be extended further. The 4-site plaquette is the smallest subunit that allows resonance between pairs of dimers and that keep the fundamental point group symmetry of the square lattice, the $C_{4}$ axis. The same requirements naturally lead to the 12 -site diamond cluster for the triangular lattice: A 4-site plaquette does not have $C_{3}$ symmetry, and the 6-site plaquette that has the shape of a large triangle does not allow pairs of dimers facing each other. So one can conjecture that there is a general tendency for such models, when the dimer repulsion is no longer strong enough to stabilize the fully resonating state, to develop local RVB order in symmetric unit cells that allow sufficient resonance.

Finally, although we know quite a lot by now about the QDM on the triangular lattice, a number of very interesting questions remain, among which the role of temperature, the nature of the excitation spectrum in the RVB phase, and the possible role of visons at the transition to the ordered phase.

\section{ACKNOWLEDGMENTS}

We thank S. Korshunov for discussions on some symmetry related aspects of the problem. This work was supported by the Swiss National Fund and by MaNEP. F.B. and M.F. are partially supported by CNR-INFM and by MIUR (COFIN-2004). F.B. thanks EPFL for the kind hospitality during the accomplishment of this work.
${ }^{1}$ P. W. Anderson, Science 235, 1196 (1987).

${ }^{2}$ G. Misguich and C. Lhuillier, in Frustrated Spin Models, edited by H. T. Diep (World Scientific, New Jersey, 2004).

${ }^{3}$ D. S. Rokhsar and S. A. Kivelson, Phys. Rev. Lett. 61, 2376 (1988).

${ }^{4}$ F. Mila, Phys. Rev. Lett. 81, 2356 (1998).

${ }^{5}$ M. E. Zhitomirsky, Phys. Rev. B 71, 214413 (2005).

${ }^{6}$ F. Vernay, A. Ralko, F. Becca, and F. Mila, Phys. Rev. B 74, 054402 (2006).

${ }^{7}$ R. Moessner and S. L. Sondhi, Phys. Rev. Lett. 86, 1881 (2001).

${ }^{8}$ A. Ralko, M. Ferrero, F. Becca, D. Ivanov, and F. Mila, Phys. Rev. B 71, 224109 (2005).

${ }^{9}$ D. A. Ivanov, Phys. Rev. B 70, 094430 (2004).

${ }^{10}$ In the present paper, we only study the gap in the excitations generated by the dimer density operator ("non-vison-like" excitations in the terminology of Ref. 9). The "visonlike" sector of excitations has a different gap and will be the subject of subsequent studies. Note that in a finite system visonlike excitations are possible only in pairs, and therefore the visonlike spectrum is not visible in conventional finite-system calculations. On the other hand we believe that, in the non-vison-like sector, the dimer density operator generally has nonvanishing matrix elements with the lowest excitations, and therefore that our calculations give the correct value of the gap for those excitations. We have verified this explicitly for small systems up to 36 sites by comparing with exact-diagonalization data.

${ }^{11}$ N. Trivedi and D. M. Ceperley, Phys. Rev. B 41, 4552 (1990).

${ }^{12}$ M. Buonaura Calandra and S. Sorella, Phys. Rev. B 57, 11446 (1998).

${ }^{13}$ L. Spanu, F. Becca, and S. Sorella, Phys. Rev. B 73, 134429 (2006).

${ }^{14}$ S. Sorella and L. Capriotti, Phys. Rev. B 61, 2599 (2000).

${ }^{15}$ J. E. Gubernatis, M. Jarrell, R. N. Silver, and D. S. Sivia, Phys. Rev. B 44, 6011 (1991).

${ }^{16}$ B. Bernu, P. Lecheminant, C. Lhuillier, and L. Pierre, Phys. Rev. B 50, 10048 (1994).

${ }^{17}$ R. Moessner and S. L. Sondhi, Phys. Rev. B 63, 224401 (2001).

${ }^{18} \mathrm{~S}$. Korshunov (private communication). 